\title{
NUVENS ESCURAS AUSTRAIS. I - CONTAGEM DE ESTRELAS E EXTINÇŌES VISUAIS
}

\author{
GILBERTO CARLOS SANZOVO \\ MARIA ALCINA BRAZ
}

\begin{abstract}
RESUMO
Neste trabalho, o método de contagem de estrelas desenvolvido por Dickman (1976) foi aplicado a 3 nuvens escuras do Hemisfério Celeste Sul, com o objetivo de determinar a sua extinção visual, $A_{V}$. As nuvens foram selecionadas a partir dos Atlas ESO (B), SRC-J e ESO (R) e as contagens feitas em 2 campos distintos: o campo da nuvem escura e o campo de comparação, livre de obscurecimento. A partir dos valores determinados de $A_{V}$, vários glóbulos escuros foram reconhecidos e suas dimensões estimadas.
\end{abstract}

PALAVRAS-CHAVE: Nuvens escuras; Extinções visuais; Contagem de estrelas.

\section{INTRODUÇÃO}

As nuvens escuras interestelares são facilmente reconhecidas nas placas fotográficas pelo obscurecimento que causam, provocando uma diminuição aparente na densidade de estrelas nas regiões onde se localizam. Essas regiões são relativamente frias $\left(\mathrm{T}_{\mathrm{K}} \sim 10 \mathrm{~K}\right)$, constituídas de hidrogênio molecular e grãos de pó, que absorvem e espalham a radiação estelar incidente.

Elas são classificadas em Grandes Complexos de Nuvens Escuras, Grandes Glóbulos e Pequenos Glóbulos (BOK, CORDWELL e CROMWELL, 1971) e essa classificação leva em conta fundamentalmente sua extensão e grau de opacidade. Quanto à distribuição espacial, os Catálogos de LINDS 1962 e FEITZINGER \& STÚWE 1984 mostram que até $1 \mathrm{Kpc}$ existem muitas nuvens escuras, com forte concentração na direção do plano galático. No entanto, os catálogos existentes fornecem apenas as opacidades desses objetos, sendo necessária a utilização de métodos estatísticos, como contagem de estrelas para determinar a extinção visual $A_{V}$ com relativa precisão e boa resolução angular. Uma das vantagens da utilização de contagem de estrelas para determinar $A_{V}$ é a possibilidade de obter uma resolução angular tal que pontos centrais (mais obscurecidos) das nuvens sejam distinguidos e comparados, ponto a ponto, com os resultados de outras observações realizadas em diferentes comprimentos de onda.

Em 1974, JENKINS e SAVAGE obtiveram uma razão constante entre o $\mathrm{H}$ molecular e a extinção visual para regiões em nuvens escuras com $A_{V^{\sim}} 3$ mag. Com argumentos teóricos fortemente conclusivos, DICKMAN (1978) estende a aplicabilidade dessa "razão gás-pó" para pontos centrais mais obscurecidos nas nuvens estudadas ( $A_{V^{\sim}} 10$ mag) dinamizando, com isso, o uso de seu método e permitindo um estudo mais completo dos traçadores químicos nesses objetos.

\section{O MÉTODO DE DICKMAN}

O método de DICKMAN (1976) envolve contagem de estrelas sem a preocupação de se fazer uma divisão em intervalos de magnitudes aparentes. A contagem do número total de estrelas é realizada em pequenos elementos quadrados ("reseau") de uma rede, construída em papel acetato. Deve-se tomar cuidado na escolha da fronteira que separa a nuvem escura escolhida do campo de comparação, pois este preferencialmente não deve sofrer a influência obscurecedora da nuvem. Após terem sido escolhidos os campos, fixa-se a rede sobre a placa fotográfica que contém a região a ser estudada e procede-se à contagem, uma a uma, de todas as estrelas em cada elemento da rede para ambos os campos. As contagens são realizadas sobre uma mesa adequadamente preparada para tal finalidade e com o auxílio de um microscópio.

Como as placas fotográficas são sensitivas às cores azuis e vermelhas - com bandas passantes de comprimentos de onda médios $\mathrm{B}\left(\lambda_{\mathrm{B}^{\sim 4}} 4300 \AA\right), \mathrm{J}\left(\lambda_{\mathrm{J}^{\sim 4}} 4700 \AA\right)$ e $\mathrm{R}\left(\lambda_{\mathrm{R}^{\sim 6500}} \AA\right)$, as extinções determinadas nestes comprimentos de onda serão proporcionais ao número de estrelas contadas no campo de comparação e no campo da nuvem. Assim, se n( *) representa o número de estrelas contadas no campo de comparação e $n(O)$ o número delas no campo da nuvem, as extinções $A_{B}, A_{J}$ e $A_{R}$ valem, respectivamente

a. Departamento de Física - CCE/UEL

b. Departamento de Astronomia - IAG/USP 


$$
\begin{aligned}
& A_{B}=\frac{1}{b_{B}} \log \frac{n_{B}(*)}{n_{B}(O)} \quad \text { mag } \\
& A_{J}=\frac{1}{b_{J}} \log \frac{n_{J}(*)}{n_{J}(O)} \quad m a g \\
& A_{R}=\frac{1}{b_{R}} \log \frac{{ }_{R}{ }^{(*)}}{{ }^{n}{ }_{R}(O)} \quad m a g
\end{aligned}
$$

onde $b_{B^{-}} b_{J}-b_{R}$ são constantes a serem determinadas (VAN RHIJN, 1929; DICKMAN, 1978).

A redução dessas extinções para o seu equivalente padronizado $\mathrm{A}_{\mathrm{V}}$, com comprimento de onda médio $\lambda_{\mathrm{V}} \sim 5500 \AA$ é simplificada se são conhecidas a lei de avermelhamento local $f\left(\lambda^{-1}\right)$ e a razão $R$ entre as extinçoes total e seletiva, de modo que

$$
A_{V}=\frac{R}{R+f\left(\lambda^{-1}\right)} A_{\lambda} \text { mag }
$$

Para as regiões consideradas neste trabalho, tomando o valor $\mathrm{R}=3,2$ (HARRIS, 1973) e os valores de $\mathrm{f}\left(\lambda_{\mathrm{B}}-1\right) \sim 1$, $\mathrm{f}\left(\lambda_{\mathrm{J}}\right) \sim 0,65$ e $\mathrm{f}\left(\lambda_{\mathrm{R}}\right) \sim-0,55$ da curva de avermelhamento de WHITFORD (1958), a Equação 4 transforma-se em

$$
\begin{array}{ll}
\mathrm{A}_{\mathrm{V}}=0,76 \mathrm{~A}_{\mathrm{B}} & \mathrm{mag} \\
\mathrm{A}_{\mathrm{V}}=0,83 \mathrm{~A}_{\mathrm{J}} & \mathrm{mag} \\
\mathrm{A}_{\mathrm{V}}=1,21 \mathrm{~A}_{\mathrm{R}} & \mathrm{mag}
\end{array}
$$

que fornecem as extinções visuais a partir das extinções no azul e vermelho.

Dickman aplicou seu método para determinar $A_{V}$ em nuvens escuras do Hemisfério Celeste Norte. Recentemente o método foi aplicado por CERNICHARO \&BACHILLER (1984) para a obtenção de um catálogo de nuvens e glóbulos escuros localizados nos Complexos de Touro e Perseu, enquanto que SANZOVO (1976) aplicou o mesmo método para nuvens escuras austrais.

\section{PROCEDIMENTO EXPERIMENTAL}

O seguinte procedimento foi utilizado para a obtenção das extinções:

a) Escolha das regiơes a partir da inspeção visual das Placas ESO, levando-se em consideração sua importância e localização no Hemisfério Celeste Sul;

b) Construção da rede de contagem com elementos unitários de forma geométrica conveniente. Com o objetivo de comparar os resultados aqui obtidos com futuras observações em ondas de rádio, padronizou-se uma rede retan- gular com elementos quadrados de $2 \times 2 \mathrm{~mm}$, o que corresponde a uma resolução angular de 2,25;

c) Para os locais onde não se contou nenhuma estrela, normalizou-se toda a área para uma única subdivisão da rede de contagem, com a finalidade de estimular um limite inferior para as extinções visuais. Para que as contagens fossem realizadas nos mesmos locais mas nos diferentes atlas ESO, elas foram inicialmente obtidas das placas azuis $B$ delimitando-se, cada área contada e marcando, na rede de contagem, posições referentes a algumas estrelas com coordenadas previamente conhecidas do Catálogo SAO. A mesma área foi, então, localizada nas placas azuis $\mathrm{J}$ correspondentes (no caso de Vela, também em R), pela sobreposição das estrelas anteriormente marcadas na rede. Procedendo dessa maneira, a incerteza no posicionamento de cada elemento da rede é sempre menor que $30 \%$ da resolução usada. Essa incerteza foi confirmada através da comparação das coordenadas de alguns objetos jovens (de Herbig-Haro) e do posicionamento das estrelas tidas inicialmente como referências para cada campo;

d) Para as contagens nas placas azuis B, o valor da constante $b_{B}$, em cada nuvem escura, foi determinado a partir das Tabelas de VAN RHIJN (1929) aplicando-se o método dos mínimos quadrados. As extinçōes $A_{B}$ foram determinadas com o auxílio de (1);

e) Para as placas azuis $J$ e vermelhas $R$, os valores de $\mathrm{b}_{\mathrm{J}}$ e $\mathrm{b}_{\mathrm{R}}$ foram considerados idênticos aos de $\mathrm{b}_{\mathrm{B}}$, levando-se em consideração uma precisão de $20 \%$, descrita por DICKMAN (1978). As extinções $A_{j}$ e $A_{R}$ foram determinadas com o auxílio das Equaçoes 2 e 3 ;

f) A redução às equivalentes extinções visuais foram feitas com o auxílio das Equações 5, 6 e 7.

\section{AS REGIÔES ESCOLHIDAS E OS RESULTADOS OB- TIDOS}

As regiões estudadas foram escolhidas devido a suas localizações nos Hemisfério Celeste Azul e por apresentarem, algumas delas, objetos jovens, provavelmente associados a processos de formação de estrelas de baixa massa. A escolha foi feita através da inspeção visual de alguns campos fotográficos dos Atlas do Observatório Sul Europeu (ESO), localizado em La Silla, Chile.

Foram selecionadas nuvens escuras nas direçoes do Saco de Carvão Sul, Circinos e Vela. Os resultados sao representados na forma de matrizes - matrizes contagens e matrizes extinções. Os mapas são dispostos em ordem crescente de numeração dos Campos ESO e as configuraçð̃es são tais que a direção Norte localiza-se no topo e Leste à esquerda de cada figura. Os principais resultados obtidos são descritos a seguir, para cada região estudada:

\section{Saco de Carvão Sul}

Uma região de 1 grau quadrado, contendo pouco mais de 16000 estrelas, foi estudada no Saco de Carvão Sul. A região aparece na Placa ESO(B) 095 e o resultado das contagens é mostrado na Figura 1. Uma visðo superficial do mapa é suficiente para identificar 4 glóbulos escuros na nuvem. A região foi estudada por TAPIA (1973) e sua 
distância estimada em 175 pc por RODGERS (1959), obtida através de medidas fotométricas, no sistema UBV, de 1060 estrelas distribuídas homogeneamente na sua direção. A Figura 2 mostra a distribuição da extinção visual e a localização de 32 fontes de infravermelho distantes (IRAS,1985). $O$ gradiente em $A_{V}$ é acentuado nas proximidades do maior glóbulo da figura onde, para uma distância angular relativamente pequena, correspondente a $\sim 2,25, A_{V}$ varia em quantidade superior a 3,2 mag. Fazendo uma pesquisa da distribuição do $\mathrm{H}$ atômico para a região, BOWERS, KERR e HAWARDEN (1980) deduziram $\mathrm{A}_{\mathrm{V}}=6,6$ mag. para a região central mais densa da nuvem escura, o que está de acordo com os resultados aqui apresentados.

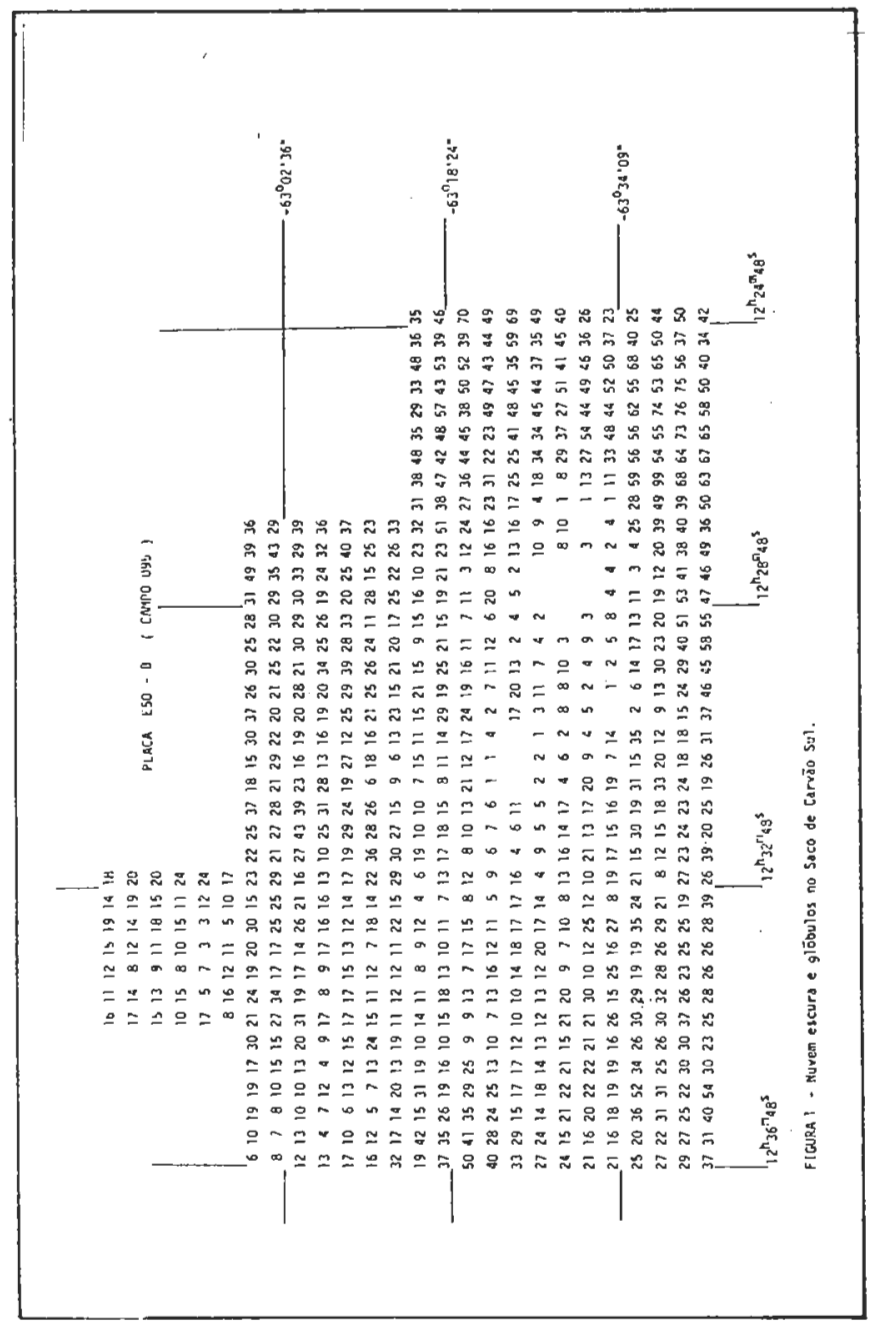

FIGURA 1 : Mostra a matriz contagem de estrelas (Placa B) n nuvem escura e glóbulos no Saco de Carvão Sul

\section{Circinos}

Região de pequenas dimensões angulares $(\sim 13 ; 7 \times 7,5)$, muito obscurecida e envolvida em um rico campo com distribuição uniforme de estrelas (Figura 3). Ela é facilmente visível na direção Norte da Placa ESO(B) 098. O mapa de (iso) extinções visuais é mostrado na Figura 4. Apenas uma fonte infravermelha foi identificada na regiåo, que apresenta um glóbulo associado à nuvem escura. 0 glóbulo, compacto e esférico, possui uma área equivalente a $0,07 \mathrm{pc}^{2}(\sim 0,15 \mathrm{pc}$ de raio) e está localizado à distância de 450 pc. Essa distância foi deduzida por DANZIGER et alii (1975) por meio de espectrofotometria de uma estrela próxima a uma nebulosa de reflexăo localizada a 4,5 (a Leste) do glóbulo e coincidente com a posiçáo da fonte IRAS. Através de contagem de estrelas esses autores encontraram uma extinção $A_{B}=6,5$ mag para a região central do glóbulo. As curvas de iso-extinçбes mostram que $A_{V}$ decresce rapidamente do centro do glóbulo para as bordas da nuvem. Na direção Norte, o decrescimo é mais pronun. ciado; para uma distância angular correspondente a 4,5 $\mathrm{A}_{\mathrm{V}}$ diminui de um valor superior a $2,7 \mathrm{mag}$, o que reflete bem o isolamento e a compactabilidade do glóbulo.

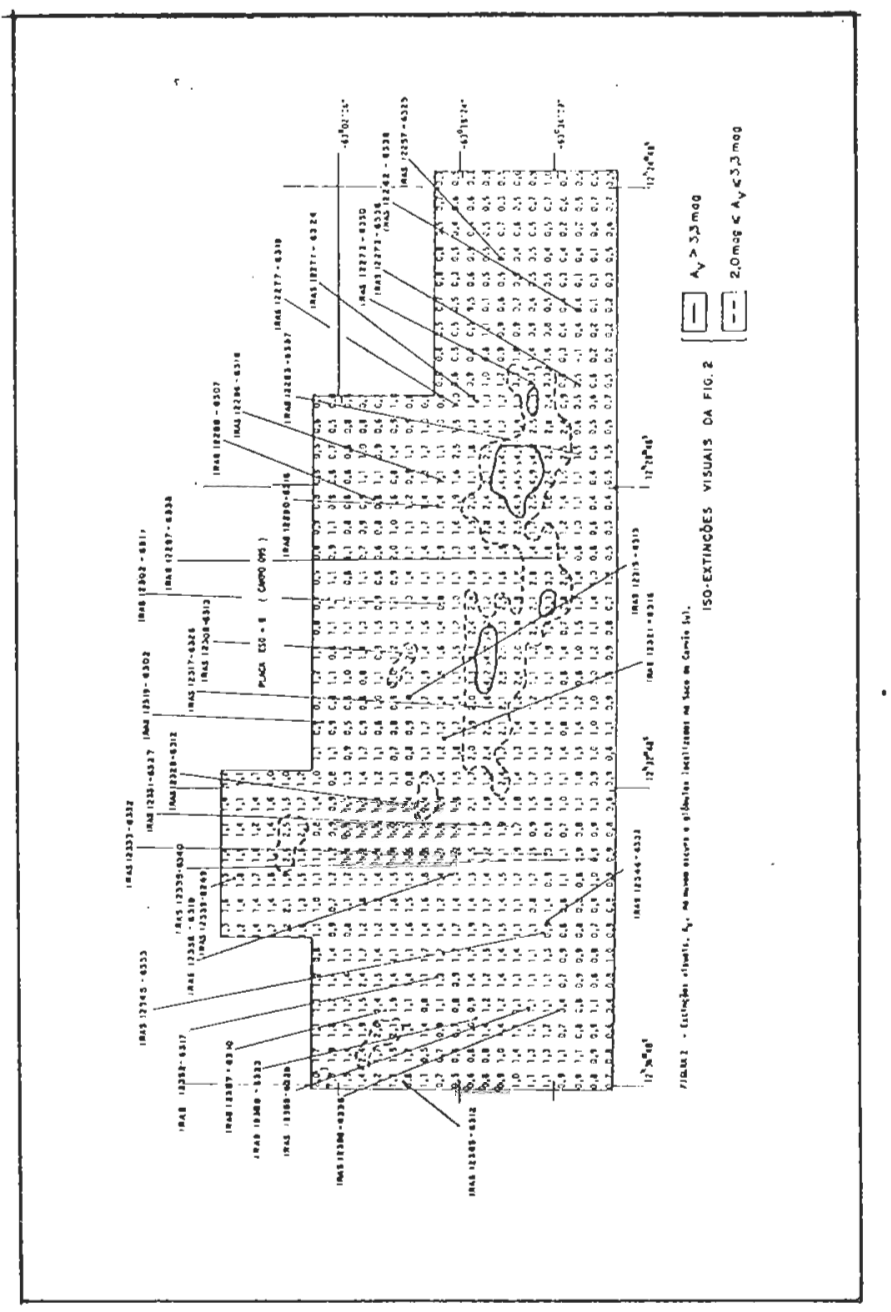

FIGURA 2: Mostra a matriz de extinçōes visuais (Placa B) na nuvem escura e glóbulos localizados no Saco de Carvão Sul. Várias fontes infravermethas IRAS foram identificadas na região

\section{Vela}

Em Vela, as contagens realizaram-se nas Placas azuis B e $J$ e vermelha $R$ do Campo ESO 210. As Figuras 5, 6 e 7 mostram as matrizes contagens e extinções para as placas $B$ e R. As curvas de iso-extinções visuais destacam partes centrais com $A_{V}>2,0$ mag e um glóbulo escuro. Outras regiões menores também aparecem contornadas e suas presenças são explicadas pela não uniformidade na distribuição do obscurecimento pela nuvem escura. O glóbulo 


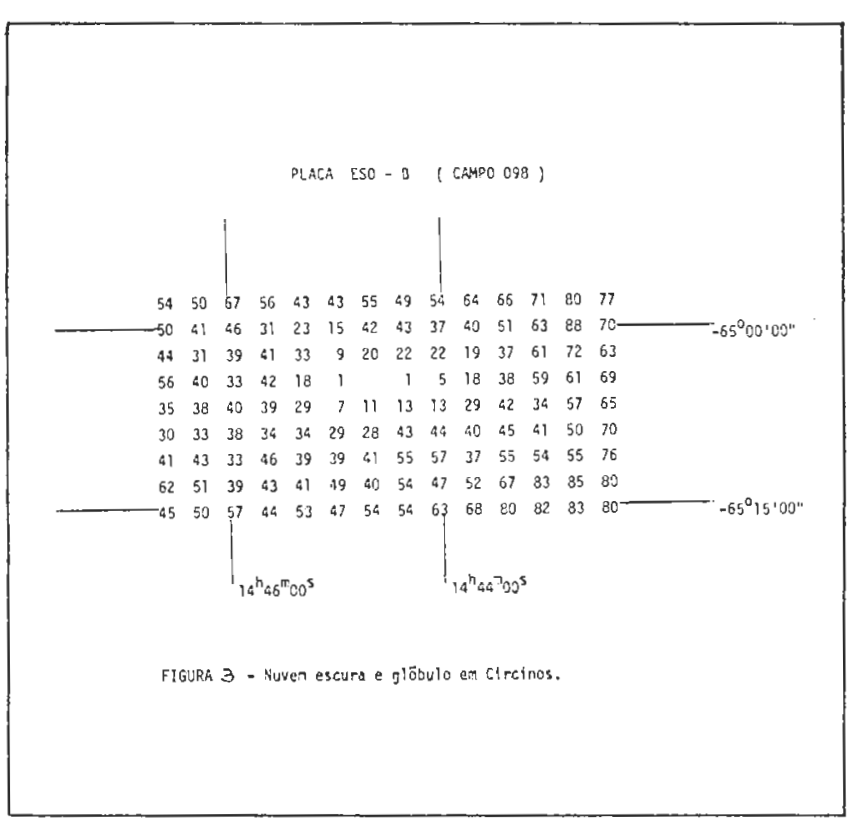

FIGURA 3: Mostra a matrix contagem de estrelas (Paca B) para a nuvem escura e glóbulo em Circinos

é conhecido na Literatura como Glóbulo de Bok ESO 210 $6 \mathrm{~A}$ e apresenta 2 objetos jovens próximos à sua extremidade Norte, associados com a emissão infravermelha distante (IRAS 1985). Esses objetos de Herbig-Haro foram descobertos por SCHWARTZ (1977) durante a realização de uma pesquisa de estrelas $\mathrm{H}_{\alpha}$ no Hemisfério Celeste Sul. O glóbulo é visto a Oeste do mapa de iso-extinçðes visuais e possui uma dimensão angular de $~ 2,3$ o que corresponde a um diâmetro de $\sim 0,2$ pc para a distância de 300 pc proposta por SCHWARTZ (1977). É muito compacto e isolado e apresenta $A_{V}>5,2 \mathrm{mag}$, na placa $R$. No Catálogo de FEITZINGER \& STUWE (1984) ele aparece classificado como Objeto Globular 51 com opacidade variando entre 5 e 6.

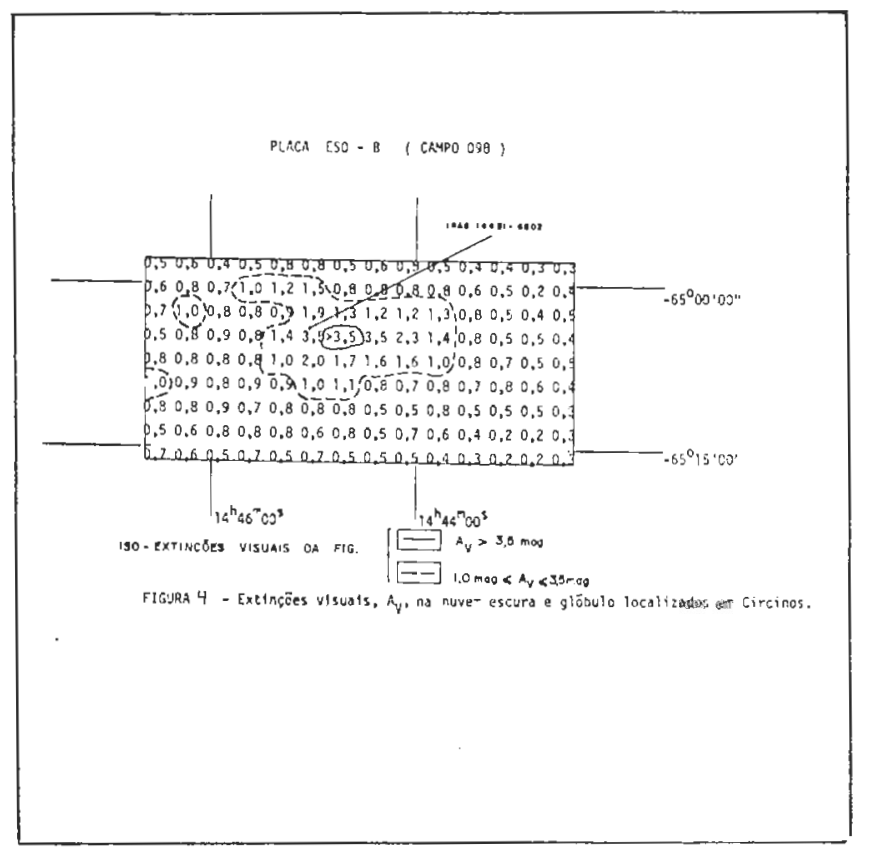

FIGURA 4: Mostra a matriz de extinções visuais para a nuvem escura em Circinos

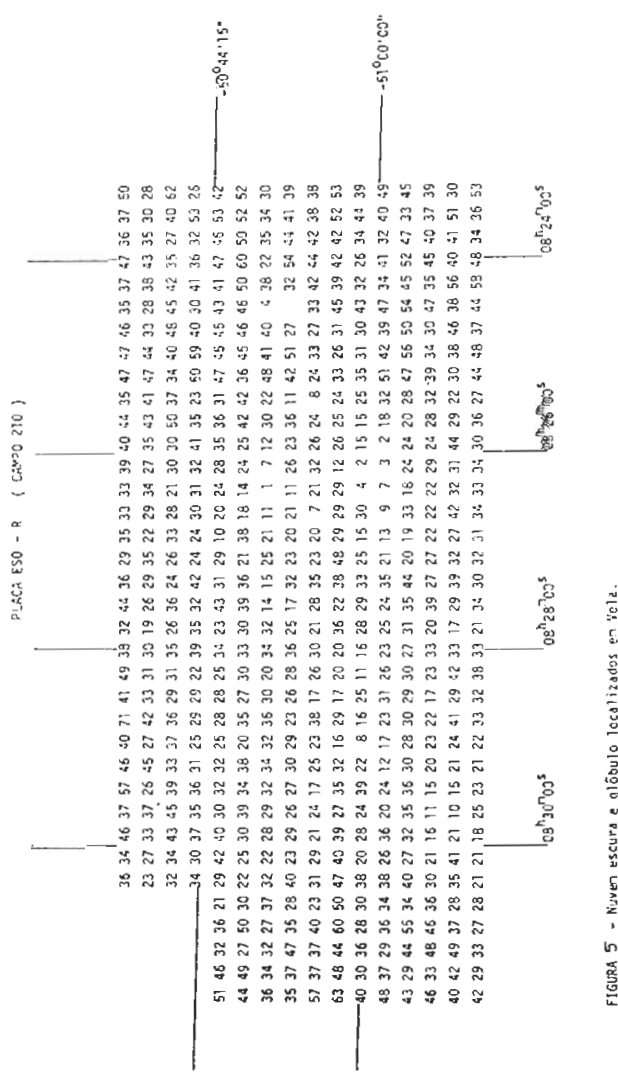

FIGURA 5: Mostra a matriz contagem de estrelas (Placa R) para a nuvem escura e glóbulo localizados em Vela.

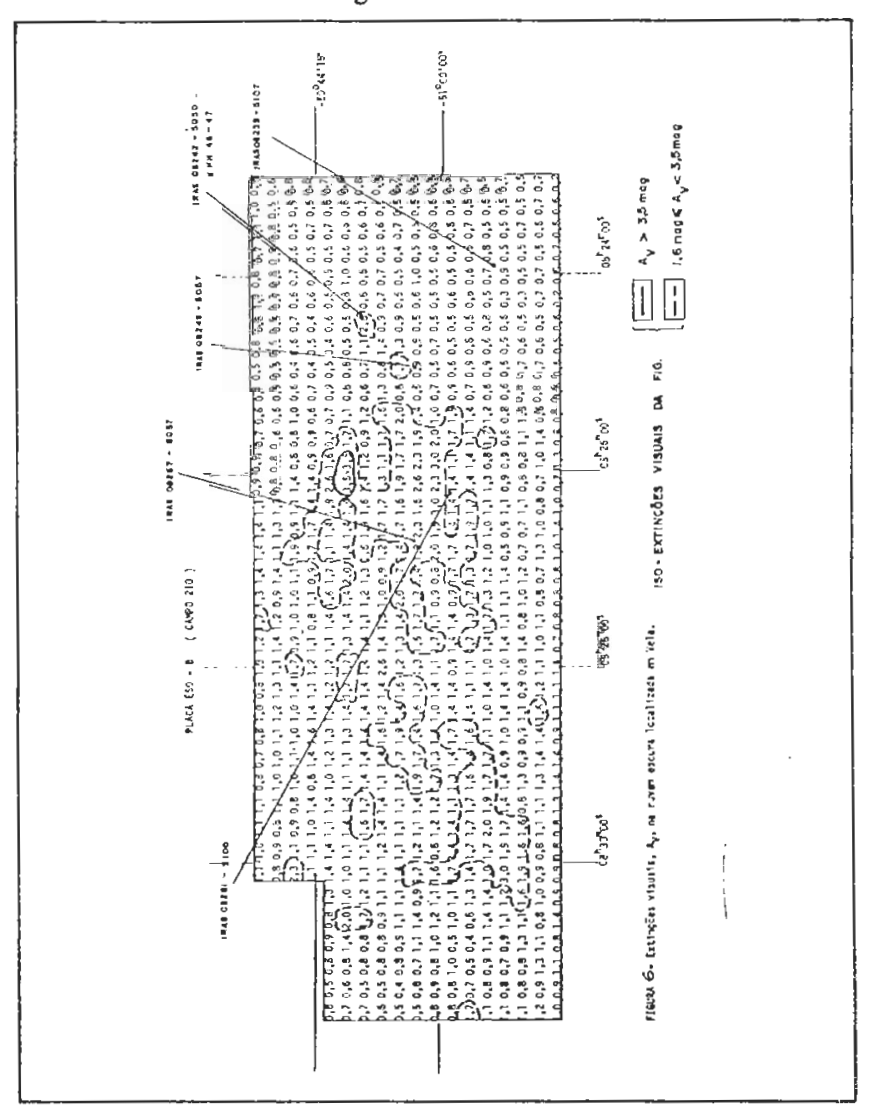

FIGURA 6: Mostra a matriz extinçōes visuais (Paca B) para a nuvem escura e glóbulo localizadas em Vela 


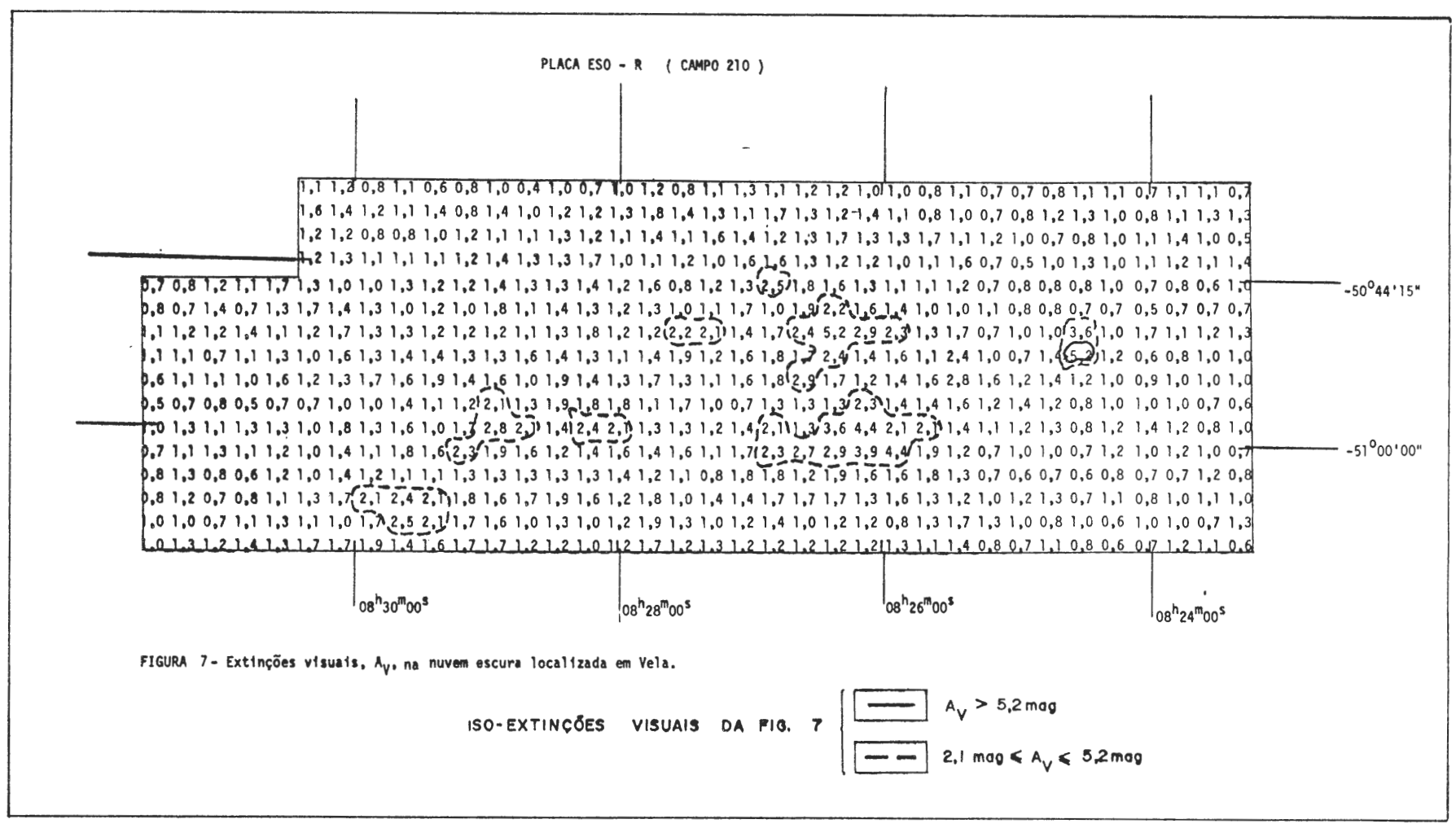

FIGURA 7: Mostra a matriz extinções visuais (Placa R) para a nuvem escura e glóbulo localizadas em Vela

\section{CONCLUSÕES}

Os métodos que envolvem contagem de estrelas são trabalhosos mas à medida que se aplicam técnicas modernas tornam-se simples e precisos. O objetivo de se contar estrelas é, geralmente, determinar o seu número por intervalo de magnitudes visuais no interior da placa fotográfica analisada. O método aplicado neste trabalho não envolveu tal divisão: contou-se estrelas sem a preocupação de se fazer a divisão em intervalos de magnitudes aparentes, devido ao baixo número delas nas regiões escolhidas. O método revelou-se uma ferramenta eficaz na determinadação da extinção visual das nuvens escuras, permitindo, ainda, a indentificação de vários glóbulos escuros - objetos provavelmente ligados à fomação estelar de baixa massa.

\section{ABSTRACT}

Star counts of 3 southern dark clouds were performed using the techniques developed by Dickman (1976) in order to determine the visual extinction, AV. Using the ESO (B), SRC-J and ESO (R) plates, counts were made in the cloud anc' comparison fields, the latter being presumably free of obscurity. Various globules were identified in the clouds and parameters as dimension and visual extinction were estimated.

KEY-WORDS: Dark clouds; Visual extinctions; Star counts.

\section{REFERENCIAS BIBLIOGRÁFICAS}

01. BOK, B.J., CORDWELL, C.S. e CROMWELL, R.H. Dark Nebulae, Globules and Protostars, eds. B.T. Lunds. Tucson, University of Arizona Press, 1971.

02. BOWERS, P.F., KERR, F.J. e HAWARDEN, T.G. Astrophys . $J ., 241: 1980$.

03. CERNICHARO, J. \& BACHILLER, R. Astron. Astrophys. Suppl. Ser., 58:327, 1984.

04. DANZIGER, I.J., DENNEFELD, M., HAVLEN, R.J. e
SCHUSTER, H. - E. Astron. Astrophys., 40:455, 1975.

05. DICKMAN, R.L. PhD. Dissertation (Colum bia University), 1976.

06. - - - Astron. J. 83: 363, 1978.

07. FEITZINGER, J.V. \& STÜWE, J.A. Astron. Astrophys. Suppl. Ser. 58: 365, 1984. 
09. IRAS Catalogs and Aulases. 1985, eds. C.A. Beichamn, G. Neugebauer, H.J. Habing, P.E. Qlegg e T.J. Chester.

10. JENKINS, E.B. \& SAVAGE, B.D. Astrophys, J. 187: 243, 1974.

11. LYNDS, B.T. Astrophys. J. Suppl. 7:, 1, 1962.

12. RODGERS, A.W. Monthly Notices Roy. Astron. Soc. 120: $163,1959$.
13. SANZOVO, G.C. em "Estudo de Nuvens Escuras Austrais", Dissertação de Mestrado, USP, 1986.

14. SCHWARTZ, R.D. Astrophys. J. Suppl. 35: 161, 1977.

15. TAPIA, S. IAU Symp. n. 52, 1973.

16. VAN RHIJN, P.J. Groninger Publ. n. 43, 1929.

17. WHITFORD, A.E. Astron. J. 63:, 201, 1958. 